\title{
Prevalencia del juego patológico en Galicia mediante el NODS. ¿Descenso de la prevalencia o mejor evaluación del trastorno?
}

\author{
Elisardo Becoña \\ Facultad de Psicología. Universidad de Santiago de Compostela \\ Enviar correspondencia a: \\ Elisardo Becoña. Universidad de Santiago de Compostela. Facultad de Psicología. Departamento de Psicología Clínica y Psicobiología. Campus Universitario Sur. \\ 15782 Santiago de Compostela. E-mail: pcelisar@usc.es. \\ Recibido: 4 de noviembre de 2003. \\ Aceptado: 26 de junio de 2004.
}

\section{RESUMEN}

El juego patológico constituye actualmente un importante problema de salud mental que acarrea un gran número de problemas a nivel personal, familiar y social. En el presente estudio se analiza la prevalencia del juego patológico en Galicia, en una muestra representativa de los adultos de 18 o más años ( $N$ = 1.624), recogida en 33 municipios, estratificada por hábitat, edad y sexo. Se utilizó para le evaluación del juego patológico, juego problema y juego en riesgo el NODS (NORC DSM-IV Screen for Gambling Problems) de Gernstein et al. (1999). Los 17 ítems del NODS se agrupan en las diez categorías del DSMIV para el diagnóstico de juego patológico (5 o más criterios).

Los resultados indican que un $0.92 \%$ serían jugadores patológicos a lo largo de su vida, y un $0.31 \%$ a lo largo del último año; un $0.18 \%$ jugadores problemas a lo largo de toda la vida y $0.25 \%$ para el último año, y un $0.31 \%$ de jugadores de riesgo a lo largo de toda la vida y $0.25 \%$ para el último año. Estos resultados son claramente inferiores a los obtenidos en otros estudios previos en Galicia y en España, pero equivalentes a los norteamericanos de validación del NODS. Se discute la necesidad de adecuarse a los criterios del DSM-IV, y a instrumentos derivados del mismo, en los estudios de prevalencia del juego patológico. Igualmente se presentan datos de otros estudios recientes que permiten explicar los menores niveles de prevalencia actuales que los obtenidos hace unos años. Los criterios más restrictivos del DSM-IV, y los cuestionarios derivados del mismo, permitirían explicar el menor nivel de prevalencia actual, junto a que hoy existe una mayor concienciación social sobre los problemas que acarrean los juegos de azar.

\section{ABSTRACT}

At the present time, pathological gambling is an important mental health problem that carries with it a large number of problems at a personal, family and social level. This study analyses the prevalence of pathological gambling in Galicia (NW Spain), in a representative sample of adults of 18 years of age or above ( $N=1.624$ ), collected in 33 municipalities, stratified by habitat, age and sex. The NODS (NORC DSM-IV Screen for Gambling Problems) by Gernstein et al. (1999) was used for the assessment of pathological, problem and at-risk gamblers. The 17 NODS items cover the ten DSM-IV categories for the diagnosis of pathological gambling ( 5 or more criteria).

The results indicate that $0.92 \%$ are pathological gamblers throughout their lifetime, and $0.31 \%$ in the past year; $0.18 \%$ problem gamblers throughout their lifetime and $0.25 \%$ in the past year; and $0.31 \%$ are at risk of becoming gamblers throughout their lifetime and $0.25 \%$ in the past year. These results are clearly lower those obtained in other previous studies in Galicia and Spain, but equivalent to the North America NODS evaluation. We discuss the necessity of adjustment to the DSM-IV criteria, and to instruments derived from the same, in studies on the prevalence of pathological gambling. Data is also presented from other recent studies that permit an explanation for the current prevalence levels being lower than those obtained some years ago. The more restrictive DSM-IV criteria, and the questionnaires derived from it, would explain the lower level of current prevalence, in addition to a greater social awareness, nowadays, on the problems of gambling.

Key words: NODS, pathological gambling, prevalence, Spain.

\section{INTRODUCCIÓN}

E n pocos años el juego patológico se ha convertido en un problema de gran relevancia social y con una clara repercusión clínica. Desde su apa- rición formal en el DSM-III (American Psychiatric Association, 1980) hasta nuestros días, se han realizado un gran número de estudios para conocer su prevalencia en la población, como sus características clínicas, psicopatológicas y tratamiento para el mismo. 
El juego de azar se ha legalizado en España en 1977, como ocurrió en torno a aquellos años en otros países desarrollados, y las máquinas tragaperras en 1981. En pocos años fueron apareciendo en el mercado los distintos juegos con un enorme crecimiento del gasto en juego y el incremento del número de jugadores que participan en los distintos juegos de azar. En España los juegos más populares son las loterías, cupones y máquinas tragaperras, siendo éstas últimas, junto al bingo, las que tienen el mayor poder de adicción (Becoña, 1996a). Actualmente, el gasto en juego en España es, en los últimos datos disponibles para el año 2002, de 25.853,84 millones de euros (4,3 billones de las antiguas pesetas), con un gasto per cápita de 628,82 euros por habitantes, aunque con significativas diferencias de gasto en juego de unas a otras comunidades autónomas (Comisión Nacional del Juego, 2003). Por órden de importancia de gasto en juego están: máquinas tragaperras, bingo, loterías (Lotería Nacional), loterías de organismos benéficos (ONCE), lotería primitiva y bonoloto, y casino. Tampoco debemos olvidarnos de que, de los países de la Comunidad Económica Europea, ocupamos los primeros puestos de gasto per cápita en juego (Becoña, 1996b).

Actualmente disponemos de un gran número de estudios epidemiológicos sobre la prevalencia del juego patológico en distintos países, como en España, tanto realizados en adultos como en niños y adolescentes. Así, en adultos, hay estudios sobre su prevalencia en Canadá (Ferris y Wynne, 2001; Ladouceur, 1991, 1996), Nueva Zelanda (Abbott y Volberg, 1996, 2000), Australia (Dickerson, Baron, Hong y Cottrell, 1996), Reino Unido (Sproston, Erens y Orford, 2000), Suecia (Rönnberg, 2000), etc. Pero donde más se han realizado estudios de prevalencia ha sido en Estados Unidos. La National Research Council (1999) recoge 33 estudios epidemiológicos y, más recientemente, la Alberta Gaming Research Institute (www.abgaminginstitute.ualberta.ca), un total de 52, realizados a lo largo de los distintos estados norteamericanos. Muchos de ellos son estudios de replicación realizados con varios años de diferencia de unos a otros en el mismo estado. La mayoría de estos estudios han sido realizados utilizando el South Oaks Gambling Screen (SOGS) de Lesieur y Blume $(1987,1993)$ para evaluar el juego patológico, destacando que ha sido Rachel Volberg la que ha llevado a cabo una buena parte de los mismos en Estados Unidos. Hasta los estudios realizados en los últimos años, la prevalencia estimada de juego patológico en Estados Unidos, para el período del último año, no solía bajar del 1\% Ilegando incluso al 2\% y más (ver Volberg, 1993, 1996, 2002).

En España se han hecho varios estudios para conocer la prevalencia del juego patológico con muestras representativas de distintas comunidades autóno- mas, destacando los estudios en Galicia (ej., Becoña, 1993a, 1993b; Becoña y Fuentes, 1995) y en Andalucía (Irurita, 1996; Ramírez et al., 1999). En ellos se ha utilizado el South Oaks Gambling Screen (SOGS) o cuestionarios derivados del DSM-III, DSM-III-R o DSM-IV. Con estos instrumentos la prevalencia oscila entre el $1.4 \%$ y el $1.9 \%$ (Becoña, 1999), con un buen nivel de consistencia a lo largo de los distintos estudios para evaluar los "probables jugadores patológicos". En cambio ha sido menos consistente para la evaluación de los "probables jugadores problema", la cual es una categoría no clínica, o subclínica, de la que en ocasiones se ha abusado en exceso. Esta categoría tiene un claro interés para la investigación y para conocer los perfiles sociológicos de los tipos de jugadores, desde el que no juega en los juegos de azar hasta el que tiene problemas clínicos por dicho juego. Pero lo cierto es que se ha abusado con mucha frecuencia de esta denominación, y de los resultados de los estudios donde aparece esta denominación, sumandose incorrectamente en más de un estudio los jugadores patológicos a los jugadores problema como si nos estuviésemos refiriendo a lo mismo.

En adolescentes la prevalencia es mayor que en los adultos, normalmente del doble al triple (ej., Fisher, 1993), resultado que se encuentra consistentemente en la mayoría de los países, como en España (ej., Becoña, Míguez y Vázquez, 2001a, 2001b). En adolescentes suele hablarse de juego problema más que de juego patológico, utilizándose instrumentos derivados del SOGS o del DSM-IV. Sin embargo, como ocurre actualmente con los resultados en adultos, estos datos pocas veces tienen un claro correlato con el diagnóstico clínico siguiendo los criterios del DSM-IV (Winters, 2000).

Pero el cuadro anterior está cambiando a un ritmo muy rápido en los últimos años. En 1994 se publica el DSM-IV con nuevos criterios diagnósticos para el juego patológico, que se mantienen idénticos en el DSM-IV-TR (American Psychiatric Association, 2000). Vienen a ser una combinación de algunos criterios del DSM-III, otros del DSM-III-R, junto al criterio de escape, o utilización del juego como escape de las situaciones cotidianas, tales como ansiedad, depresión, etc. (Lesieur y Rosenthal, 1991). Al mismo tiempo, se considera que para llegar al diagnóstico de juego patológico hay que descartar un episodio maníaco (no sería jugador patológico, por ejemplo, aquel consumidor de cocaína que juega descontroladamente después del consumo de cocaína, pero cuando no consume cocaína no juega).También destaca que de 9 criterios del DSM-III-R se pasa a 10 en el DSM-IV y el punto de corte sube de 4 a 5 criterios del DSM-III-R al DSM-IV. Para los estudios epidemiológicos estos cambios son de una gran relevancia porque el instrumento que se venía utilizando predominantemente para evaluar el juego patológico era el SOGS, el cual 
es una combinación del DSM-III y DSM-III-R, pero ya no se adecua completamente al DSM-IV. Por ello, en los últimos años varios autores (ej., Abbott y Volberg, 1996; Ferris y Wynne, 2001; Lesieur, 2002; National Research Council, 1999; Stinchfield, 2002; Volberg, 1999) han indicado el problema de los falsos positivos con el SOGS. Más específicamente, Stinchfield (2002) concluye como en la población general el SOGS sobreestima la prevalencia del juego patológico en el doble, ya que en su estudio encuentra que un $50 \%$ de sus casos son falsos positivos. Esto indica que con el SOGS la prevalencia es mucho mayor que la que encontraríamos de utilizar los criterios diagnósticos del DSM-IV, criterios que son los que tenemos que utilizar para hablar o no de juego patológico, porque es nuestro referente.

Dado que los datos anteriores sugieren que se ha sobreestimado la prevalencia del juego patológico en la población general, tanto en otros países como en el nuestro, en 1999 un grupo de expertos norteamericanos, algunos de los más relevantes del juego patológico en ese país, elaboran un nuevo instrumento, el NODS (NORC DSM-IV Screen for Gambling Problems), basado en los criterios del DSM-IV (Gernstein, Murphy, Tace, Hoffmann, Palmer, Johnson et al., 1999). La idea de elaborar este instrumento no es otra que la de disponer de un sistema de evaluación del juego patológico fiable, adecuada a los criterios del DSM-IV y que pueda servir para comparar unos estudios con otros. Con el mismo se realizó un amplio estudio con una muestra representativa norteamericana, donde encontraron un $0.8 \%$ de jugadores patológicos para el período de toda la vida y un $0.1 \%$ de jugadores patológicos en el último año, y sin diferencias por sexo (en hombres $0.9 \%$ y $0.1 \%$ para vida y último año, y para mujeres de $0.7 \%$ y $0.2 \%$ para vida y último año). Lo cierto es que estos resultados son muy, pero que muy inferiores, a los datos que se estaban manejando sobre la prevalencia del juego patológico en Estados Unidos, entre el 1\% y el 2\% de la población adulta (Volberg, 1996, 2002). El porcentaje de jugadores problema (3 ó 4 criterios) también es inferior a estudios previos, encontrando un $1.3 \%$ para la vida y un $0.4 \%$ para el último año. Donde las cifras son muy altas es en la categoría de jugadores en riesgo, que son aquellos con una puntuación de 1 ó 2 criterios en el NODS, ya que en este caso hay un $7.9 \%$ de personas en riesgo a lo largo de la vida y un $2.3 \%$ en el último años y donde aparecen también claras diferencias por sexos $19.6 \%$ y $3.2 \%$ en hombres para vida y último año y $6.3 \%$ y $1.6 \%$ para esas mismas categorías en mujeres). Pero esto puede ser engañoso, porque esta denominación de juego en riesgo nada tiene que ver con criterios diagnósticos del DSM-IV y puede continuar, de no saber bien a qué nos referimos con esta categoría, a incrementar la confusión si se abusa de este término.
Lo cierto es que, por lo dicho hasta ahora, el NODS es un instrumento de evaluación del juego patológico que solventa algunos de los problemas de instrumentos previos, como el SOGS, y se basa en los criterios del DSM-IV, que son los criterios de referencia que tenemos que seguir actualmente para hablar o no de juego patológico. Además, los datos obtenidos con el NODS sobre especificidad y sensibilidad son buenos y el instrumentos se ha derivado directamente de los criterios diagnósticos del DSM.-IV, con criterios estrictos y de alta calidad a nivel metodológico y de representatividad de las muestras utilizadas para su validación. Su fiabilidad test-restest es de 0.98 y su validez es excelente al adecuarse estrictamente a los criterios del DSM-IV.

Dado que en España se han realizado varios estudios epidemiológicos representativos con varias poblaciones con distintos instrumentos de evaluación del juego patológico basados en el SOGS, DSM-III y DSM-III-R, pero ninguno con un instrumento validado directamente a partir del DSM-IV, y además validado siguiendo una metodología precisa y consistente, y con una muestra representativa para una validación adecuada, el objetivo del presente estudio es conocer la prevalencia del juego patológico en una muestra representativa de la población gallega utilizando el NODS, instrumento que se adecua a los criterios diagnósticos del DSM-IV para el juego patológico.

\section{MÉTODO}

\section{Muestra}

La muestra utilizada está compuesta por 1.624 personas de 18 o más años representativa de la población gallega (760 varones y 864 mujeres). La muestra se obtuvo aleatoriamente en 36 municipios gallegos, seleccionados al azar, mediante un muestreo aleatorio estratificado proporcional. Los estratos fueron el hábitat (urbano -las sietes ciudades gallegas-, intermedio -municipios de 10.001 a 50.000 habitantes- y rural -aquellos municipios con menos de 10.000 habitantes), el sexo y la edad (18-30, 31-45, 46-64 y 65 o más años). El porcentaje de rechazos para el total de la muestra fue del $8.4 \%$. La muestra tiene un error muestral máximo del 2,5\% para un intervalo de confianza del $95.5 \%$.

La distribución por edades de la muestra, que se adecuaba a la distribución de las mismas en la población, fue la siguiente: 336 tenían entre 18 y 30 años, 389 entre 21 y 45 años, 474 entre 46 y 64 años y 425 65 o más años. Cuatrocientas tres personas estaban solteras, 971 casadas, 189 viudos, 48 separados o 
divorciados y 13 vivían en pareja. De la zona urbana eran 584, de la intermedia 469 y de la rural 571.

Las personas fueron seleccionadas aleatoriamente en sus domicilios, utilizando un sistema de rutas aleatorias en cada punto muestral previamente prefijado por el investigador para localizar cada casa.

\section{Instrumentos de evaluación}

Se utilizó un cuestionario en el que se incluían datos demográficos, datos relacionados con su conducta de juego y distintas escalas psicométricas, entre ellas el NODS (NORC DSM-IV Screen for Gambling Problems) de Gernstein et al. (1999) para la evaluación del juego pagológico. Este instrumento consta de 17 ítems que se agrupan en las diez categorías del DSM-IV para el diagnóstico de juego patológico (5 o más criterios) (ver tabla 1). El mismo diferencia a los jugadores patológicos (5 o más criterios) de los jugadores problema (3 ó 4 criterios) y jugadores en riesgo (1 ó 2 criterios). A su vez con el NODS evaluamos los problemas de juego a lo largo de la vida como en el último año. Es necesario, antes de poder considerar darle una puntuación en el NODS a una persona, que hayan jugado y gastado dinero en juegos de azar. En el estudio norteamericano de validación utilizaron el criterio de que hubiesen perdido 100 dólares aunque sólo haya sido en un sólo día en el año, o que al menos hayan jugado un total de 100 dólares en el trascurso de un año. En nuestro caso se considera que para que a una persona le sean aplicables los criterios del NODS, tiene que haber jugado en el último año un mínimo de 15.000 pts.(año 2001) [90 euros].

\section{Procedimiento}

La aplicación de los cuestionarios para este estudio fue realizada por seis psicólogos especialmente entrenados para el mismo. Los encuestadores partían de un punto muestral prefijado de antemano en cada lugar (ej., aldea de una parroquia) de cada municipio, y se seleccionaba cada casa o piso con una cadencia fija de cómputo. Seleccionada la casa o piso donde se iba a realizar la encuesta se seleccionaba a la persona a encuestar aleatoriamente, en función de los estratos de edad y sexo.

Las entrevistas fueron personales, en el domicilio de las personas. Cuando la persona no estaba en su domicilio se acudió en distintos días y horas para localizar a la misma. En los casos en los que no fue posible localizarla se consideró como no respuesta, añadiéndose al cómputo final de rechazos del estudio.

Las entrevistas fueron realizadas en los meses de marzo a julio de 2001.
Los entrevistadores, psicólogos como ya hemos indicado, llegaban, si era el caso, al diagnóstico de juego patológico mediante el NODS. Si éste era el diagnóstico pasaban a continuación de la aplicación del cuestionario a realizar con la persona una entrevista clínica específica para jugadores patológicos (ver Becoña, 1993a) que permitía confirmar el diagnóstico y obtener datos de su historia y características de juego. Con posterioridad otras dos personas, independientes del entrevistador, analizaban toda la información recogida para confirmar o no el diagnóstico realizado. Estos casos aparecían entremezclados con otros de jugadores problema, en riesgo o sin problemas de juego. En caso de duda o discrepancia se utilizaba el criterio más conservador respecto a la implicación de la persona en el juego.

\section{RESULTADOS}

Del total de los sujetos de la muestra, para el periodo de toda la vida, con el NODS nos encontramos que el $98.56 \%(n=1.601)$ reconoce que no tiene problemas con el juego. Los que puntúan 5 o más en el NODS, y siempre que jueguen un mínimo de 15.000 pts. al año, serían jugadores patológicos, los de 3-4 jugadores problema y los de 1-2 jugadores en riesgo. Los resultados indican que un $0.92 \%$ ( $n=$ 15) serían jugadores patológicos a lo largo de su vida, y un $0.31 \%$ ( $n=5)$ a lo largo del último año. En todos los casos estas personas son varones. A su vez, el porcentaje de jugadores problemas fue, para toda la vida, de un $0.18 \%(n=3)$, y de un $0.25 \%(n=4)$ para el último año. El porcentaje de los jugadores de riesgo fue de un $0.31 \%(n=5)$ para toda la vida y de un $0.25 \%(n=4)$ para el último año. La agrupación de estas dos últimas categorías nos indicaría un 0.49\% $(n=8)$ de jugadores problema y de riesgo tanto para el periodo de toda la vida como del último año. Hay que notar que existen diferencias en los mismos individuos para estar en unas o en otras categorías. Por ejemplo, varios jugadores patológicos en la vida pueden estar en la categoría actual de no jugadores, jugadores problema o jugadores de riesgo. Como en el caso de los jugadores patológicos, en todos los casos de los jugadores problema y de riesgo en esta muestra son hombres, sin ninguna mujer (ver tabla 1).

El análisis de las respuestas de toda la muestra a los distintos ítems del NODS nos indica que hay un porcentaje de respuestas afirmativas a los mismos semejante tanto para la prevalencia de vida como para la del último año (ver tabla 2), cubriendo los criterios básicos del DSM-IV para el juego patológico, especialmente los primeros ítems y, en menor grado, los últimos, que son los del mayor nivel de gravedad. 
Los ítems más importantes, por orden, han sido los siguientes: "ha intentado alguna vez dejar, reducir o controlar el juego", "En una o más de estas ocasiones de intentos de dejar, reducir o controlar el juego se sentió intranquilo o irritable", "ha tenido períodos de 2 o más semanas en las que pasó una gran cantidad de tiempo pensando en sus experiencias con el juego o planificando futuros episodios de juego o de apuestas", "alguna vez ha intentado dejar, reducir o controlar su conducta de juego sin poder conseguirlo", "ha tenido períodos de 2 o más semanas en los que pasó mucho tiempo pensando en cómo conseguir dinero para jugar", "ha tenido alguna vez un período en el cual si perdía dinero en el juego volvía otro día para recuperarlo", "ha tenido períodos de 2 o más semanas en los que necesitaba jugar con cantidades de dinero cada vez mayores o apuestas mayores que antes para conseguir la misma excitación", "ha mentido alguna vez a su familia, amigos o a otros sobre cuánto juega o cuánto dinero perdía en el juego", y "alguna vez ha intentado dejar, reducir o controlar su conducta de juego sin poder conseguirlo". En la tabla 3 presentamos los ítems para el total de la muestra agrupados por las 10 categorías del DSM-IV para el diagnóstico de juego patológico. Los cinco ítems más relevantes, por órden, para el período de a lo largo de la vida, son los de: preocupación, recuperación com- pulsiva de las pérdidas, síndrome de abstinencia, tolerancia y pérdida de control.

Más específicamente, para los jugadores patológicos y jugadores problema y en riesgo, en la tabla 4 presentamos los resultados más relevantes obtenidos por ellos en distintas variables sociodemográficas. Por el escaso tamaño de la muestra para el último año, presentamos los resultados para la vida, agrupando a su vez a los jugadores problema con los jugadores en riesgo. De este modo, por sexo, el $100 \%$ de los jugadores patológicos y jugadores problema y en riesgo son varones. Por edades, el 20\% de los jugadores patológicos tienen de 18 a 30 años, el $33.3 \%$ de 31 a 45 años, el $6.7 \%$ de 46 a 64 años y el $40 \% 65$ o más años. En los jugadores problemas y en riesgo el $37.5 \%$ tiene de 18 a 30 años y un $50 \%$ de 46 a 64 años. Por estado civil, en los jugadores patológicos predominan los casados, un $60 \%$, junto a los solteros, un $26.7 \%$. En los jugadores problemas y en riesgo el $50 \%$ son solteros y el $37.5 \%$ casados. Por hábitat, el $20 \%$ de los jugadores patológicos son urbanos, el $40 \%$ viven en municipios intermedios y el restante $40 \%$ en municipios rurales. En los jugadores problema y en riesgo hay igualmente un $25 \%$ en la zona urbana y rural y el restante $50 \%$ en la zona intermedia.

Tabla 1. Porcentajes de jugadores patológicos y jugadores problemas o de riesgo mediante el NODS (NORC DSM-IV Screen for Gambling Problems), en la muestra de Galicia (España) (2001) y la de validación del NODS de E.E.U.U. (1999). Prevalencia de vida y del último año.

\begin{tabular}{|c|c|c|c|c|}
\hline \multirow[b]{2}{*}{ Prevalencia (NODS) } & \multicolumn{2}{|c|}{ GALICIA (ESPAÑA) } & \multicolumn{2}{|c|}{ E.E.U.U. } \\
\hline & Vida & Último año & Vida & Último año \\
\hline \multicolumn{5}{|c|}{ Juego patológico (5 o más items) } \\
\hline - Todos & 0.92 & 0.31 & 0.8 & 0.1 \\
\hline - Hombre & 0.92 & 0.31 & 0.9 & 0.1 \\
\hline - Mujer & 0.00 & 0.00 & 0.7 & 0.2 \\
\hline \multicolumn{5}{|c|}{ Juego problema (3 ó 4 items) } \\
\hline - Todos & 0.18 & 0.25 & 1.3 & 0.4 \\
\hline - Hombre & 0.18 & 0.25 & 1.6 & 0.4 \\
\hline - Mujer & 0.00 & 0.00 & 1.0 & 0.4 \\
\hline \multicolumn{5}{|c|}{ Juego de riesgo (1 ó 2 items) } \\
\hline - Todos & 0.31 & 0.25 & 7.9 & 2.3 \\
\hline - Hombre & 0.31 & 0.25 & 9.6 & 3.2 \\
\hline - Mujer & 0.00 & 0.00 & 6.3 & 1.6 \\
\hline \multicolumn{5}{|c|}{ Agrupación de juego problema y de riesgo } \\
\hline - Todos & 0.49 & 0.49 & 9.2 & 2.7 \\
\hline - Hombre & 0.49 & 0.49 & 11.2 & 3.6 \\
\hline - Mujer & 0.00 & 0.00 & 7.3 & 2.0 \\
\hline
\end{tabular}


Tabla 2. Respuestas afirmativas en los ítems del NODS (muestra total, $\mathrm{N}=1.624$ )

A lo largo En el útlimo

de su vida año

1. ¿Ha tenido períodos de 2 o más semanas en las que pasase una gran cantidad de tiempo pensando en sus experiencias con el juego o planificando detalladamente futuros episodios de juego o de apuestas?

2. ¿Ha tenido períodos de 2 o más semanas en los que pasase mucho tiempo pensando en cómo conseguir dinero para jugar?

3. ¿Ha tenido períodos de 2 o más semanas en los que necesitaba jugar con cantidades de dinero cada vez mayores, o apuestas mayores que antes, para conseguir la misma excitación?

4. ¿Ha intentado alguna vez dejar, reducir o controlar su juego?

5.En una o más de estas ocasiones de intento de dejar, reducir o controlar su juego, ¿se sintió intranquilo o irritable?

6. ¿Alguna vez ha intentado dejar, reducir o controlar su conducta de juego sin poder conseguirlo?

7.En el caso de que fuese así, ¿ha sucedido 3 o más veces?

8. ¿Ha jugado alguna vez como una forma de escapar de los problemas personales?

9. ¿Ha jugado alguna vez para aliviar sentimientos desagradables como culpabilidad, ansiedad, indefensión o depresión?

10. ¿Ha tenido alguna vez un período en el cual si perdía dinero en el juego volvía otro día para recuperarlo?

11. ¿Ha mentido alguna vez a su familia, amigos o a otros sobre cuánto juega o cuánto dinero perdía en el juego?

12.Si es así, ¿esto ha sucedido 3 o más veces?

13. ¿Ha extendido alguna vez un cheque sin fondos o cogido dinero que no era suyo de familiares $u$ otra persona para gastar en el juego?

14. ¿Le ha causado alguna vez el juego problemas graves o repetidos en su relación con algún familiar o amigo?

15. ¿Le ha producido el juego algún problema con los estudios, como por ejemplo perder clases o días de escuela o suspender algún curso?

16. ¿Le ha causado el juego la pérdida de un trabajo, tener problemas en el trabajo o no poder aprovechar una oportunidad profesional importante?

17. ¿Ha necesitado alguna vez pedir dinero prestado a un familiar, o a otra persona, para poder salir de una situación económica desesperada causada en gran parte por su juego? 
Tabla 3. Puntuación positiva en los ítems agrupados del NODS (muestra total, $N=1,624$ )

A lo largo En el útlimo

de su vida año

1. Preocupación (¿Ha tenido períodos de 2 o más semanas en las que pasase una gran cantidad de tiempo pensando en sus experiencias con el juego o planificando detalladamente futuros episodios de juego o de apuestas?; o ¿Ha tenido períodos de 2 o más semanas en los que pasase mucho tiempo pensando en cómo conseguir dinero para jugar?)

2. Tolerancia (¿Ha tenido períodos de 2 o más semanas en los que necesitaba jugar con cantidades de dinero cada vez mayores, o apuestas mayores, que antes para conseguir la misma excitación?)

3. Síndrome de abstinencia ( $\mathrm{HHa}$ intentado alguna vez dejar, reducir o controlar su juego?; y En una o más de estas ocasiones de intento de dejar, reducir o controlar su juego, ¿se sintió intranquilo o irritable?)

4. Pérdida de control (¿Alguna vez ha intentado dejar, reducir o controlar su conducta de juego sin poder conseguirlo?; y En el caso de que fuese así, ¿ha sucedido 3 o más veces?)

5. Escape ( ¿Ha jugado alguna vez como una forma de escapar de los problemas personales?; O ¿Ha jugado alguna vez para aliviar sentimientos desagradables como culpabilidad, ansiedad, indefensión o depresión?)

6. Recuperación compulsiva ( ¿Ha tenido alguna vez un período en el cual si perdía dinero en el juego volvía otro día para recuperarlo?)

7. Mentir (¿Ha mentido alguna vez a su familia, amigos o a otros sobre cuánto juega o cuánto dinero perdía en el juego?; y Si es así, ¿esto ha sucedido 3 o más veces?)

8. Actos ilegales ( $¿ \mathrm{Ha}$ extendido alguna vez un cheque sin fondos o cogido dinero que no era suyo de familiares u otra persona para gastar en el juego?)

9. Arruinar relaciones significativas (¿Le ha causado alguna vez el juego problemas graves o repetidos en su relación con algún familiar o amigo?; o ¿Le ha producido el juego algún problema con los estudios, como por ejemplo perder clases o días de escuela o suspender algún curso?; o ¿Le ha causado el juego la pérdida de un trabajo, tener problemas en el trabajo o no poder aprovechar una oportunidad profesional importante?

10. Mantenimiento financiero ( $\mathrm{iHa}$ necesitado alguna vez pedir dinero prestado a un familiar, o a otra persona, para poder salir de una situación económica desesperada causada en gran parte por su juego?) 
Tabla 4. Características sociodemográficas de los jugadores patológicos, jugadores problema y jugadores de riesgo (prevalencia de vida).

\begin{tabular}{|lccc|}
\hline & $\begin{array}{c}\text { Jugador } \\
\text { patológico } \\
\text { (N = 15) }\end{array}$ & $\begin{array}{c}\text { Jugador problema } \\
\text { o en riesgo } \\
\text { (N = 8) }\end{array}$ & $\begin{array}{c}\text { Sin problemas } \\
\text { de juego } \\
\text { (N = 1601) }\end{array}$ \\
\hline Sexo & & & \\
- Hombre & 100.0 & 100.0 & 46.0 \\
- Mujer & 0.0 & 0.0 & 54.0 \\
Edad & & & \\
- 18-30 & 20.0 & 37.5 & 20.6 \\
- 31-45 & 33.3 & 0.0 & 24.0 \\
- 46-64 & 6.7 & 50.0 & 29.3 \\
- 65+ & 40.0 & 12.5 & 26.1 \\
Estado civil & & & \\
- Soltero & & 50.0 & 24.7 \\
- Casado/pareja & 26.7 & 37.5 & 60.7 \\
- Separado/divorciado & 60.0 & 12.5 & 2.9 \\
- Viudo & 0.0 & 0.0 & 11.7 \\
& 13.3 & & \\
Hábitat & & 25.0 & 36.2 \\
- Urbano & & 50.0 & 35.7 \\
- Intermediio & 20.0 & 25.0 & 35.2 \\
- Rural & 40.0 & & \\
\hline
\end{tabular}

\section{DISCUSIÓN}

Los resultados obtenidos en este estudio con el NODS (NORC DSM-IV Screen for Gambling Problems) muestran niveles de prevalencia claramente inferiores a los que hasta el momento se han indicado en estudios previos en España, y más concretamente en Galicia, sobre el juego patológico. Así, se encuentra un $0.9 \%$ de jugadores patológicos a lo largo de la vida y un $0.3 \%$ en el último año. Estos resultados son claramente inferiores a los obtenidos en otros estudios con instrumentos distintos, como ocurría con el SOGS, tanto en Galicia (Becoña y Fuentes, 1995) como en otras comunidades autónomas (ej., Ramírez et al., 1999), como utilizando otros instrumentos o metodologías (ej., Becoña, 1993a; Irurita, 1996; Ramírez et al., 1999). Por ejemplo, los estudios previos realizado en Galicia, indicaban un prevalencia del $1.7 \%$ de jugadores patológicos y de un $1.6 \%$ de jugadores problemas, utilizando los criterios del DSM-III-R (Becoña, 1993a, 1993b) y, con el SOGS, del 1.4\% de jugadores patológicos y un $2.0 \%$ de jugadores problema (Becoña y Fuentes, 1995). En el primer estudio la muestra era representativa de la población adulta de las siete ciudades de Galicia y en el segundo de toda la población adulta de Galicia.

Sin embargo, los resultados obtenidos en este estudio van en la línea de los obtenidos en norteamérica cuando se ha utilizado el mismo instrumento. Así, en la muestra representativa de Estados Unidos para la validación del NODS se obtuvo una prevalencia de jugadores patológicos del $0.1 \%$ para el último año y del $0.8 \%$ para la prevalencia de vida (Gernstein et al., 1999) (ver tabla 1). En cambio el porcentaje para los jugadores en riesgo o problema, obtenidos en el presente estudio con una muestra representativa de Galicia, es mucho más baja que la obtenida en el estudio norteamericano, que fue del $9.2 \%$ para la vida y del $2.7 \%$ en el último año (en la de Galicia del $0.5 \%$ y $0.5 \%$, respectivamente, para la vida y último año). Esto indica que mientras que la prevalencia de juego patológico es semejante en España y en Estados Unidos, en cambio hay una clara diferencia en lo que atañe a la prevalencia de vida y en el último año para el juego problema y para el juego en riesgo. También, hay que notar que en Estados Unidos llevan más décadas jugando con apuestas a los juegos de azar que en España. 
También es destacable que en otros estudios se han encontrado en estos últimos años niveles de prevalencia menores que hace pocos años. En el caso de Nueva Zelanda, en este último caso utilizando una versión revisada del SOGS (Abbott y Volberg, 2000), la prevalencia del juego patológico para el último año era del $0.5 \%$, cuando hace años, para su estudio de 1991, encontraron prevalencias del 1.2\% de jugadores patológicos para el momento actual y del $2.7 \%$ para la vida (Abbott y Volberg, 1996). Esta prevalencia es también casi la misma que la encontrada recientemente en Suecia, del 0.6\% para el último año, con el SOGS (Rönnberg, 2000). En ambos casos no encuentran una clara explicación para este hecho. Igualmente, en Canadá, con un nuevo instrumento para evaluar el juego patológico, el Canadian Problem Gambling Index (CPGI) (Ferris y Wynne, 2001), encontraron una prevalencia del $0.7 \%$, que con el SOGS subía al $1.3 \%$. La diferencia aquí está en que un instrumento se adecúa a los criterios actuales del DSM-IV (el CPGI) y en el otro (el SOGS) en parte a los del DSM-III y DSM-III-R. Por tanto, hoy ya podemos afirmar, en función de los estudios previos, como de otros (ej., Stinchfield, 2002, 2003), y revisiones recientes (Raylu y Oei, 2002), que en la población general el SOGS sobreestima la prevalencia del juego patológico en el doble del existente con criterios del DSM-IV. O, lo que es igual, que encuentra un $50 \%$ de falsos positivos. Como sabemos hoy el DSM-IV es el criterio básico de referencia para llegar a un diagnóstico de juego patológico.

En la prevalencia de vida del presente estudio se encontraron diferencias significativas por sexo, por ser todos los jugadores patológicos varones (100\%), así como los jugadores en riesgo (100\%). Dado que es clara la existencia de mujeres jugadoras patológicas que acuden a tratamiento, el no encontrarlas en este estudio lo achacamos a la baja prevalencia encontrada en función de la muestra utilizada. No se encontraron diferencias por hábitat (urbano, intermedio, rural) ni por grupos de edad, aunque esto puede en parte ser debido al bajo número de jugadores en cada categoría.

Una posible explicación para el bajo nivel de prevalencia encontrado para el juego problema y el juego de riesgo en este estudio, en relación a otros realizados previamente, en esta misma población años atrás, puede deberse a la característica de la muestra encuestada, población gallega, representativa de toda una comunidad autónoma caracterizada por un alto nivel de ruralización, alto nivel de envejecimiento, con pocos servicios y acceso reducido a los juegos de azar más adictivos, como son las máquinas tragaperras y el bingo, que suelen centralizarse en grande urbes, o en las urbes que existen en Galicia. De este modo las ciudades tendrían mas peso, aunque los datos actuales de este estudio concreto no permiten afirmar esto de modo concluyente, aunque otro tipo de datos nos indican que el mayor problema del juego patológico se asocia al tamaño de la ciudad, como también explica el que en las ciudades haya mayor número de recursos asistenciales para los jugadores patológicos, las grandes ciudades sean los que gastan más dinero en juegos de azar por habitantes, haya mayor número de máquinas tragaperras por cada 100.000 habitantes, etc. (Becoña, 1993a, 1996; Comisión Nacional del Juego, 2003). Por ello, aunque esto está claro en otros estudios, no aparece así en este. Quizás en futuros estudios, con muestran más amplias, se pueda comprobar y confirmar o rechazar este hecho.

Igualmente el hecho del incremento de las opiniones y creencias negativas hacia el juego en Galicia (Becoña, 2002) en el momento en que se hizo el estudio (2001), respecto a datos disponibles sobre una muestra semejante 10 años atrás (1991) (Becoña, 1993a) puede explicar en parte los resultados obtenidos. Pero lo que si es cierto es que el NODS es un cuestionario mucho más restrictivo que los que hasta el momento se venían utilizando, porque los datos disponibles hasta ahora en España, como revisa Becoña (1999), iban todos en la misma línea de prevalencia, con muestras distintas, en distintas comunidades autónomas y por equipos de investigación diferentes.

Otra posible explicación para el menor nivel de prevalencia pueda venir dada porque ahora empiezan a jugar más tarde los jóvenes o lo hacen con menor intensidad. Así, el estudio de Winters (2000) ha indicado que los que eran clasificados como probables jugadores patológicos cuando eran adolescentes, a los 15-19 años, la mayoría dejaban de serlo años después, cuando tenían de 21 a 25 años, aunque en este grupo aparecían a estas edades nuevos jugadores patológicos que no lo eran antes. Esto sugiere que las altas tasas de juego problema de la niñez (ej., Becoña et al., 2001b) y de la adolescencia (ej., Becoña et al., 2001a) desaparecerán posteriormente en la adultez, de modo semejante a lo que ocurre también con el consumo de drogas y alcohol en la adolescencia respecto al fuerte descenso en la edad adulta (Bachman et al., 2002).

Otra posible explicación que se podría aducir para haberse encontrado esta baja prevalencia en relación a estudios previos es que el punto de corte de 5 criterios para el diagnóstico del juego patológico es muy alto, como varios autores han indicado (ej., Stinchfield, 2003), y que debería rebajarse a 4, utilizando el DSM-IV. Sin embargo, esto no es aplicable totalmente a nuestro estudio, porque el porcentaje de jugadores problema es también bajo. Con todo, también cabe la posibilidad de que las características de nuestros jugadores patológicos sean distintas, como indicaba Echeburúa, Báez, Fernández y Páez (1994) hace años, donde con el SOGS proponían bajar el punto de corte de 5 a 4 criterios, cuando en aquel momento el SOGS se adecuaba bastante bien a los criterios del DSM-III-R. 
Tampoco debemos dejar de lado el hecho de que el porcentaje de rechazo haya sido del $8.4 \%$, que aunque bajo para este tipo de estudios, facilita que los que tienen problemas de juego sean más reaccios a contestar al cuestionario o se nieguen a contestarlo. Los informes de campo de los entrevistadores indican en varios casos que nos podríamos encontrar con este hecho al negarse algunas personas a contestar el cuestionario al llegar a las preguntas de juego, otros negar nerviosamente que ellos jugasen, junto a la existente mayor probabilidad de negativa a la realización del cuestionario y entrevista cuando se tienen problemas psicopatológicos, entre los que puede estar el del juego patológico. Un mayor tamaño de la muestra podría solventar este hecho, aunque la utilizada era la correcta en este tipo de estudios, para el nivel de prevalencia que se había encontrado en estudio previos, en torno al $1.5 \%$.

A diferencia de estudios previos, donde se encontraba consistentemente que la prevalencia del juego patológico se daba más en hombres que en mujeres (2:1), dependía de la edad (la mayoría de los jugadores patológicos, del 30\% al 40\% tenían de 18 a 30 años), educación (los menos educados eran generalmente los más afectados) y tenían un mayor consumo de cigarrillos, alcohol y depresión (ej., Becoña, 1993a; Becoña, Fuentes y Lorenzo, 1996), este estudio no permite afirmar esto por el bajo número de jugadores patológicos. Sin embargo, la experiencia clínica y un gran número de estudios empíricos realizados (ej., Becoña, 1996; Echeburúa, Báez y Fernández-Montalvo, 1996; Fernández-Alba y Labrador, 2002; González, Jiménez y Aymamí, 1999; Rodríguez-Martos, 1989) indican que el cuadro característico de los jugadores patológicos es el que indicamos más atrás.

A pesar de todo ello, el dato del $0.3 \%$ de jugadores patológicos obtenidos en este estudio para el total de Galicia, que aunque resulta inferior al obtenido en otros estudios, indica la gravedad del problema que hemos analizado. Este porcentaje, al ser obtenido en una muestra representativa, extrapolado a toda la población de referencia, nos indicaría para las personas adultas de 18 o más años, que están en torno a 2.300.000 personas, habría 6.900 jugadores patológicos que cumplirían los criterios del DSM-IV para este trastorno para el último año. Y, a su vez habría un total de 20.700 jugadores patológicos que cumplían este criterio para el conjunto de su vida. A su vez, el número de jugadores problema o en riesgo para el último año serían otra adicional cantidad de 11.500 personas, que es la misma cifra que hay para el conjunto de su vida para la categoría de jugador problema o en riesgo. Claramente, esto representa un importante número de afectados, con los problemas que ello acarrea para ellos, sus familias y otras personas, así como para el sistema sanitario, especialmente para la asistencia en salud mental, al tener que prestarle servicio a los mismos o, al menos, a una parte de los que acuden a tratamiento para este trastorno.

El conjunto de los datos anteriores, los niveles de prevalencia encontrados, así como las características demográficas de los jugadores de este estudio, indican la necesidad de revisar los datos que hasta ahora se han manejado sobre la prevalencia del juego patológico, la necesidad de replicar este estudio y mejorar los instrumentos de evaluación del juego patológico en la población general para poder llegar a resultados consistentes en cada país y entre distintos países. Llegar a un mayor consenso de los instrumentos de evaluación de este trastorno, para la realización de estudios en la población general, es una tarea a la que hay que prestar más atención para conocer el nivel de prevalencia de este trastorno en la población.

Aunque en este estudio se ha utilizado una muestra representativa de la población adulta de Galicia, con un tamaño adecuado para este tipo de estudios ( $N=1.624)$, dados los resultados de prevalencia bajos obtenidos en la misma, la muestra viene a resultar pequeña para explotar más adecuadamente los datos, con lo que la muestra se constituye en una limitación para un mayor poder de generalización del estudio. De ahí que futuros estudios deben partir de que vamos a encontrar un nivel de prevalencia semejante a éste y por ello debe incrementarse significativamente el tamaño de la muestra. Igualmente vendría bien validar el NODS con muestras clínicas españolas de jugadores patológicos.

Como nota final, de confirmarse estos resultados en otros estudios posteriores, tendríamos que cambiar claramente el nivel de prevalencia que hasta ahora manejábamos cuando hablábamos de la prevalencia y número de jugadores patológicos en España. En 1995, Becoña, Labrador, Echeburúa, Ochoa y Vallejo (1995), en función de los datos disponibles en aquel momento en todos los estudios y evidencia empírica disponible, estimaron el número de probables jugadores patológico en España en un 1.5\%, 450.000. Si aplicásemos los datos de este estudio a aquella extrapolación la cifra anterior descendería de modo significativo. Aún así, el problema es grave en las personas afectadas y para todo el sistema social. La rigurosidad de conocer a quién afecta el problema del juego patológico y cómo les afecta no debe hacernos olvidar del enorme sufrimiento que los jugadores patológicos y sus familias sufren por este trastorno.

\section{AGRADECIMIENTOS}

Esta investigación ha sido financiada mediante el proyecto de investigación PGIDT00PXI21101PR de la Secretaría Xeral de Investigación e Desenvolvemento de la Xunta de Galicia. 
Este estudio no habría sido posible realizarlo sin el minucioso trabajo y pericia profesional de los psicólogos que recogieron los datos para este estudio, así como a todas aquellas personas que aportaron ideas y comentarios a las versiones previas de este manuscrito, como Arturo Bustelo, Sandra Garralón, Ana López, $\mathrm{M}^{a}$ Carmen Lorenzo, Loli Sánchez y Marisol Vilas.

\section{REFERENCIAS}

Abbott, M.W. y Volberg, R.A. (1996). The New Zealand National Survey of problem and pathological gambling. Journal of Gambling Studies, 12, 143-160.

Abbott, M. y Volberg, R. (2000, Junio). Prevalence of pathological gambling in New Zealand. Comunicación presentada al 11th International Conference on Gambling \& Risk Taking. Las Vegas, Nevada.

American Psychiatric Association (1980). Diagnostic and statistical manual of mental disorders, 3rd ed. Washington, D.C.: American Psychiatric Association (trad. cast. en Barcelona: Masson, 1984).

American Psychiatric Association (1994). Diagnostic and statistical manual of mental disorders, DSM-IV. Washington, D.C.: American Psychiatric Association (trad. cast., Barcelona, Masson, 1995).

American Psychiatric Association (2000). Diagnostic and statistical manual of mental disorders, DSM-IV, revised text. Washington, D.C.: American Psychiatric Association (trad. cast., Barcelona: Masson, 2002).

Bachman, J. G., O’Malley, P.M., Schulenberg, J. E., Johnston, L.D., Bryant, A. L. y Merline, A.C. (2002). The decline of substance use in young adulthood. Changes in social activities, roles, and beliefs. Mahwah, NJ: Lawrence Erlbaum Associates.

Becoña, E. (1993a). El juego compulsivo en la comunidad autónoma gallega. Santiago de Compostela: Consellería de Sanidade da Xunta de Galicia.

Becoña, E. (1993b). The prevalence of pathological gambling in Galicia (Spain). Journal of Gambling Studies, 9, 353-369.

Becoña, E. (1996a). La ludopatía. Madrid: Aguilar.

Becoña, E. (1996b). The problem and pathological gambling in Europe: The cases of Germany, Holland and Spain. Journal of Gambling Studies, 12, 179-192.

Becoña, E. (1999). Epidemiología del juego patológico en España. Anuario de Psicología, 30, 7-19.

Becoña, E. (2002). Evolución de las opiniones y creencias sobre el juego de azar en la población adulta a lo largo de 10 años: 1991-2001 [resumen]. Adicciones, 14 (Supl. 2), 94-95.

Becoña, E. y Fuentes, M.J. (1995). El juego patológico en Galicia evaluado con el South Oaks Gambling Screen. Adicciones, 7, 423-440.
Becoña, E., Labrador, F.J., Echeburúa, E., Ochoa, E. y Vallejo, M.A. (1995). Slot gambling in Spain: A new and important social problem. Journal of Gambling Studies, 11, 265-286.

Becoña, E., Lorenzo, M.C. y Fuentes, M.J. (1996). Pathological gambling and depression. Psychological Reports, 78, 635-640.

Becoña, E., Míguez, M.C. y Vázquez, F.L. (2001). El juego problema en los niños de Galicia. Madrid: Sociedad Española de Psicopatología Clínica, Legal y Forense.

Becoña, E., Míguez, M.C. y Vázquez, F.L. (2001). El juego problema en los niños escolarizados de la enseñanza secundaria de 14 y más años de Galicia. Psicothema, 13, 559-564.

Comisión Nacional del Juego (2003). Memoria de Juego 2002. Ministerio del Interior, Comisión Nacional del Juego (en www.mir.es)

Dickerson, M.G., Baron, E., Hong, S-M. y Cottrell, D. (1996). Estimating the extent and degree of gambling related problems in the Australian population: A national survey. Journal of Gambling Studies, 12, 161-178.

Echeburúa, E., Báez, C., Fernández, J. y Páez, D. (1994). Cuetionario de juego patológico de South Oaks (SOGS): Validación española. Análisis y Modificación de Conducta, 20, 769-791.

Echeburúa, E., Báez, C. y Fernández-Montalvo, J. (1996). Comparative effectiveness of three therapeutic modalities in the psychological treatment of pathological gambling: Long-term outcome. Behavioral and Cognitive Psychotherapy, 24, 51-72.

Fernández-Alba, A. y Labrador, F.J. (2002). Juego patológico. Madrid: Síntesis.

Ferris, J. y Wynne, H. (2001). The Canadian Problem Gambling Index: Final Report. En http://www.CanadianProblemGamblingindex. Final Report (22.8.2003).

Fisher, S. (1993). Gambling and pathological gambling in adolescents. Journal of Gambling Studies, 9, 277-288.

Gernstein, D., Murphy, S., Tace, M., Hoffman, J., Palmer, A., Johnson, R., et al. (1999). Gambling impact and behavior study. Chicado: Report to the National Gambling Impact Study Commission.

González, A., Jiménez, S. y Aymamí, M.N. (1999). Evaluación y tratamiento cognitivo-conductual de jugadores patológicos de máquinas recreativas con premio. Anuario de Psicología, 30, 111-125.

Irurita, I.M. (1996). Estudio sobre la prevalencia de los jugadores de azar en Andalucía. Sevilla: Comisionado para la Droga, Consejería de Asuntos Sociales.

Ladouceur, R. (1991). Prevalence estimates of pathological gamblers in Québec. Canadian Journal of Psychiatry, $36732-734$.

Ladouceur, R. (1996). The prevalence of pathological gambling in Canada. Journal of Gambling Studies, 12, 129142.

Lesieur, H.R. (2002). Epidemiological surveys of pathological gamblers: Critique and suggestions for modification. En J.J. Marotta, J.A. Cornelius y W.R. Eadington (Eds.), The downside: Problem \& pathological gam- 
bling (pp. 325-338). Reno, NV: Institute for the Study of Gambling \& Commercial Gamning.

Lesieur, H.R. y Blume, S.B. (1987). The South Oaks Gambling Screen (SOGS): A new instrument for the identification of pathological gamblers. American Journal of Psychiatry, 144, 1184-1188.

Lesieur, H.R. y Blume, S.B. (1993). Revising the South Oaks Gambling Screen in different settings. Journal of Gambling Studies, 9, 213-223.

Lesieur, H.R. y Rosenthal, R.J. (1991). Pathological gambling: A review of the literature (Prepared for the American Psychiatric Association Task Force on DSMIV Committee on Disorders of Impulse Control Not Elsewhere Classified). Journal of Gambling Studies, 7, 5-39.

National Research Council (1999). Pathological gambling: A critical review. Washington, DC: Autor.

Ramírez, J.N., Cabanes, M., Carbonero, M., Castilla, R., Duarte, J., Gutiérrez, B. et al. (1999). Análisis del sector de las máquinas recreativas "tipos $A$ y $B$ ". Estimación y análisis de la proporción de ludópatas en Andalucía. Córdoba: ANMARE.

Raylu, N y Oei, T. P. S. (2002). Pathological gambling. A comprehensive review. Clinical Psychology Review, 22, 1009-1061.

Rodriguez-Martos, A. (1989). Estudio piloto estimativo de la prevalencia del juego patológico entre los pacientes alcohólicos que acuden al programa Dross. Revista Española de Drogodependencias, 14, 265-275.

Rönnberg, S. D. (2000, Junio). Pathological gambling in Sweden. Comunicación presentada al 11th International Conference on Gambling \& Risk Taking. Las Vegas, Nevada.
Salinas, J. M. y Roa, J. (2001). Cuestionario de diagnóstico del juego patológico FAJER. Revista Internacional de Psicología Clínica y de la Salud, 1, 353-370.

Sproston, K., Erens, B. y Orford, J. (2000). Gambling behaviour in Britain: Results from the British Gambling Prevalence Survey. Londres, RU: National Centre for Social Research.

Stinchfield, R. (2002). Reliability, validity, and classification accuracy of the South Oaks Gambling Screen (SOGS). Addictive Behaviors, 27, 1-19.

Stinchfield, R. (2003). Reliability, validity and classification accuracy of a mesaure of DSM-IV diagnostic criteria for pathological gambling. American Journal of Psychiatry, 160, 180-182.

Volberg, R.A. (1993). Estimating the prevalence of pathological gambling in the United States. En W.R. Eadington y J.A. Cornelius (eds.), Gambling behavior \& problem gambling (pp. 365-378). Reno, NE: Institute for the Study of Gambling and Commercial Gaming.

Volberg, R.A. (1996). Prevalence studies of problem gambling in the United States. Journal of Gambling Studies, 12, 111-128.

Volberg, R. (1999). Research methods in the epidemiology of pathological gambling: Development of the field and directions for the future. Anuario de Psicología, 30, 3346.

Volberg, R.A. (2002). The epidemiology of pathological gambling. Psychiactric Annals, 32, 171-178.

Winters, K.C. (2000, Junio). Youth gambling: Transition from adolescence to young adulthood. Comunicación presentada al 11th International Conference on Gambling \& Risk Taking. Las Vegas, Nevada. 